\title{
Otro arquetipo femenino es posible: Interseccionalidad en Orange is the New Black
}

\author{
MHCJ no 6 | Año 2015 \\ Artículo no 11 (72) \\ Páginas 261 a 280 \\ mhjournal.org
}

\author{
Delicia Aguado Peláez (deliciaaguado@gmail.com) \\ Universidad del País Vasco - Euskal Herriko Unibertsitatea \\ Patricia Martínez García (patmartinez.garcia@gmail.com) \\ Universidad del País Vasco - Euskal Herriko Unibertsitatea
}

Palabras clave

Ficción; Interseccionalidad; Orange is the New Black; Estudios culturales; Es tudios de género; Series de televisión

\section{Sumario}

1. Introducción. 1.1 Sobre representaciones discriminatorias y op resiones entrecruzadas, 1.2. Litchfield, un escenario de diversidad femenina (y feminista). 2 . Metodología. 3. Resultados. 3.1. Dimensión I: Al teridad, 3.1.1. Mujeres aisladas en prisión. Ejes género, físico y ori entación Eje religión, 3.1.4. Ejes clase y raza. El color de piel como nuevo orden social, 3.1.4.1. 'Spanish Harlem', 3.1.4.4. 'The Others', 3.1.5. Eje edad, 3.2. Dimensión II: Otredad, 3.2.1. Nos otras vs. Sistema. 4.

Conclusiones. 5. Bibliografía. 6. Notas. sexual, 3.1.2 Eje diversidad funcional, 3.1.3. 'The Suburbs', 3.1.4.2. 'The Ghetto', 3.1.4.3.

\section{Resumen}

La calidad y la innovación de la Tercera Edad Dorada no siempre se corresponde con la ruptura de los modelos tradicionales patriarcales en los productos culturales (Aguado Peláez, 2014, 2015; Aguado Peláez y Martínez García, 2015). Sin embargo, hay producciones que van más allá, como es el caso de Orange is the New Black (Netflix, 2013).

La heterogeneidad de esta prisión federal abre las puertas a un sinfín de arquetipos femeninos. Algo que permite centrar el estudio desde una perspectiva interseccional, con el fin de comprender cómo se vive la interrelación de las diferentes desigualdades (Crenshaw, 1989; Collins, 1990). Una aplicación novedosa de dicha perspectiva al ámbito de la comunicación, ya que su práctica se ha centrado fundamentalmente en el campo de las políticas públicas (Lombardo y Verloo, 2009; Alonso, 2012; Cruells, 2015).

Así, mediante el análisis de contenido cualitativo, se plantean como objetivos: analizar 1) la identidad y las relaciones de poder en función de los ejes interseccionales; 2) las características que sustentan dichos ejes en torno a su inclusividad y variabilidad; 3) la creación de grupos sociales de referencia y el desarrollo de otredades.

En conclusión, el aglutinante identitario principal recae en la raza/etnia, justamente el eje que perpetúa más estereotipos. Por el contrario, se enfatiza la visibilización de unos sectores tradicionalmente excluidos y las estrategias para combatir las opresiones

\section{Forma de citar este artículo en las bibliografías}

Delicia Aguado Peláez y Patricia Martínez García (2015): “Otro arquetipo femenino es posible: Interseccionalidad en Orange is the New Black”, en Miguel Hernández Communication Journal, nº, páginas 261 a 280. Universidad Miguel Hernández, UMH (Elche-Alicante). Recuperado el__ de de 20__de: @link del artículo en 


\section{Another female archetype is possible: Intersectionality at Orange Is the New Black}

\author{
MHCJ no 6 | Year 2015 \\ Paperno 11 (72) \\ Pages 261 to 280 \\ mhjournal.org
}

\author{
Delicia Aguado Peláez (deliciaaguado@gmail.com) \\ Universidad del País Vasco - Euskal Herriko Unibertsitatea \\ Patricia Martínez García (patmartinez.garcia@gmail.com) \\ Universidad del País Vasco - Euskal Herriko Unibertsitatea
}

Keywords

Fiction; Intersectionality; Orange is the New Black; Cultural Studies; Gender Studies;

Television series

Sumary

1. Introduction. 1.1. About discriminatory representations and intersecting oppressions 1.2. Litchfield, a scenario of fe male diversity (and feminist). 2. Methodology. 3. Results. 3.1. Dimension I: Alterity, 3.1.1. Is olated women in prison. Axes of gender, physicala ppearance and sexual orientation, 3.1.2. Axis of functional diversity, 3.1.3. Axis of religion, 3.1.4. Axes of cl ass and race. Skin color as new social order, 3.1.4.1. 'The Suburbs', 3.1.4.2. 'The Ghetto', 3.1.4.3. 'Spanish Harlem', 3.1.4.4. 'The Others', 3.1.5. Axis of age, 3.2 . Dimension II: Otherness, 3.2.1. We vs. Sys tem. 4. Concl usions. 5. Bibliography. 6 . Notes.

\section{Abstract}

The quality and innovation of the Third Golden Age is not correlated with the break of traditional patriarchal model (Aguado Peláez, 2014;2015; Aguado Peláez y Martínez García, 2015). How ever, some productions start to break these patterns. Among them is Orange is the New Black (Netflix, 2013).

The heterogeneity of this federal prison opens up doors to a diversity of female models. Therefore, this research needs an intersectional perspective, which allows us to comprehend how the intersection of different inequalities acts (Crenshaw, 1989; Collins, 1990). The use of this perspective in Communication field is almost new, because the main focus of intersectionality has been Public Policies Analysis (Lombardo y Verloo, 2009; Alonso, 2012; Cruells, 2015).

Therefore, using the Qualitative Content Analysis, the objectives are: To analyze 1) identities and their power relationships; 2) particular characteristics of these central axes, according to their inclusiveness and variability; 3) how social groups are created and how "otherness" is developed.

In conclusion, the main binder of identity rests on race/ethnicity, precisely the axis that perpetuates more stereotypes. However, these questions do not tarnish the visibility of traditionally excluded sectors from media space or the different forms of oppression experienced by

women and their strategies to combat them.

How to cite this paper in bibliographies

Delicia Aguado Peláez and Patricia Martínez García (2015): “Another female archetype is possible: Intersectionality at Orange Is the New Black”, en Miguel Hernández Communication Journal, nº, pages 261 to 280. Universidad Miguel Hernández, UMH (Elche-Alicante). Recuperado el de de $20 \ldots$ de: [link del artículo en 


\section{Introducción}

El siglo XXI se introduce con una apuesta por la calidad y la innovación en la pequeña pantalla. Una "revolución narrativa y estética" (García Martínez, 2011: 211) que se concreta en los dramas de la Tercera Edad Dorada de la Televisión (a partir de aquí, TED). Una era mediática que se relaciona, fundamentalmente, con dos factores. Por un lado, la expansión del cable y el nacimiento de otras cadenas frente a las networks tradicionales, con el consecuente impacto en la creación y distribución de textos audiovisuales. Por otro lado, el desarrollo de tecnologías que ofrece nuevos horizontes de consumo y posibilidades de interacción entre los espectadores (Cascajosa, 2005; 2007; Tous, 2010).

Sin embargo, esta frescura narrativa no se traduce en la expansión de un modelo social menos conservador. Por el contrario, se produce una especie de reacción cultural ante el miedo reavivado durante el 11S que se concreta en el reforzamiento de las relaciones de género tradicionales y en la consecuente representación dual y jerárquica del patriarcado (Faludi, 2009; Aguado Peláez, 2014a, 2014b, 2014c, 2015) En otras palabras, las series televisivas de la TED "son usadas como vehículos de ideología de género conservadoras" (Fernández y Menéndez, 2011:3), no sólo en las expresiones más ligadas a la seguridad frente al terror, sino también en aquellas más vinculadas a la crisis que se vive a partir del 2008 y que supone, en muchos casos, una recuperación del pesimismo noir (Aguado Peláez y Martínez García, 2015). Un género que permite rescatar las figuras de anti-héroes y la población de los márgenes, así como "destruir el mito de América como espacio inclusivo" (García Martínez, 2011: 220).

En este sentido, Orange is the New Black (Netflix, 2013-), el caso de estudio de la presente investigación, ubica en pantalla a esos personajes subalternos entendidos, desde la noción de Gayatri Spivak, como aquellos pertenecientes a grupos oprimidos y silenciados (2003). Una producción que, además, se aleja de las representaciones vinculadas al tradicionalismo patriarcal que ensalza al sujeto normativo frente a todo aquel considerado 'otro'.

\subsection{Sobre representaciones discriminatorias y opresiones entrecruzadas}

Los medios de comunicación y sus ficciones se insertan en todo ese entramado institucional androcentrista que "privilegia todos los rasgos asociados a la masculinidad, mientras devalúa todo aquello codificado como femenino" (Fraser, 2011: 299). Es decir, legitiman la divisoria jerárquica entre los sexos impuesta por el contrato sexual del que habla Pateman (1995), a partir del cual se asocia lo visible "con el espacio y las tareas de los hombres blancos, heterosexuales, sin discapacidad, etc.", mientras que en el no poder se encuadran "todo el resto de sujetos que, de una forma u otra, se desvían de dicho modelo social" (Pérez Orozco, 2006: 207). 
Desde el feminismo, se ha hecho un esfuerzo por criticar y transformar la situación de múltiples discriminaciones que viven las mujeres: exclusión política, explotación económica, invisibilidad social y un largo etcétera. Un amplio abanico de desigualdades que son interiorizadas y perpetuadas a través de las representaciones culturales (Mayobre, 2007). Un ámbito en el que, en un momento de innovación tecnológica permanente, los medios de comunicación son todopoderosos exportadores de modelos sociales, también de patrones de feminidad y masculinidad.

En este sentido, y en un marco de esplendor, los dramas televisivos adquieren un papel fundamental. Estos productos, como ejemplo de expresiones culturales, son escenarios de las lógicas de dominación sociales (Young, 2000) que se conforman como fuente de violencia simbólica a partir de la ocultación y de la estereotipación de las mujeres. Un mundo de ficción donde "los varones poseen múltiples y ricas facetas y en el que se consolida un modelo de seducción apoyado en la agencia masculina frente a unas mujeres dibujadas únicamente como cuerpo para ser mirado" (Aguilar, 2001: 224).

Así, es interesante la incorporación de una perspectiva interseccional ya que permite complejizar las realidades femeninas, alejándose de representaciones homogeneizadas que perpetúan, no sólo la cosificación y la ubicación de las mujeres en la periferia, sino la invisibilización de otros grupos sociales y de existencias enredadas. El feminismo clásico se ha edificado en la denuncia de un sistema de discriminación sustentado en un sistema sexogénero. No obstante, en los últimos treinta años, se han invertido muchos esfuerzos en desarrollar un marco teórico y práctico que asumiera la existencia de identidades complejas, en las que interseccionan diferentes desigualdades (Crenshaw, 1989; 1991; Collins, 1990). Es decir, junto al género se encuentran otros sistemas de opresión por razones de raza-etnia, clase, religión, orientación sexual y edad, entre otros.

Si bien se trata de una perspectiva que es aplicada, fundamentalmente, en el campo de las políticas públicas (Lombardo y Verloo, 2009; Alonso, 2012; Cruells, 2015), se están desarrollando estudios en otros campos como el de la comunicación, que ocupa esta investigación. Y es que "media, particularly mass media such as film, television, journalism, popular fiction and all Internet-based media [...] are an important domain of intersectionality critique especially because of how they communicate and produce powerful ideas about social groups and social problems" (Grzanka, 2014: 132). Es decir, la interseccionalidad también atiende a las cuestiones de representación que se configuran para devaluar a todos aquellos colectivos considerados alteridades en la cultura popular, sean mujeres negras (Crenshaw, 1991), asiáticas (Bai, 2011), lesbianas (Platero, 2008) o de edad avanzada (Gracia, 2011).

\subsection{Litchfield, un escenario de diversidad femenina (y feminista)}

El 11 de julio de 2013 se estrena OITNB, una comedia dramática basada en el libro autobiográfico de Piper Kerman, en el que relata sus vivencias durante el año que estuvo en 
presidio. Su éxito la configura como una de las producciones estrella de la plataforma Netflix y como uno de los grandes ejemplos de la TED. Aún así, su calidad e innovación narrativa no recae tanto en la temática como en la forma de desarrollarla. Es decir, no es la primera serie centrada en mujeres en prisión -como Women in prison (Fox, 1987-1988), Bad Girls (ITV, STV, UTV, 1999-2006)-. Ni tampoco en criticar el sistema carcelario -como Oz (HBO, 1997-2003)-. Sin embargo, es de gran interés porque introduce una multiplicidad de "tabúes en la argumentación" (García y López, 2014: 21), especialmente en torno a las realidades y a la sexualidad de las mujeres. Además, esta producción ofrece la oportunidad de hablar del clasismo, la homofobia o las injusticias sociales a través de una "concatenación de contextos de discriminación" (García y López, 2014: 29).

Es definitiva, OITNB se presenta como un caso de estudio de especial interés desde una perspectiva interseccional. Pues las protagonistas se alejan de patrones simplistas vinculados a lo que tradicionalmente representa lo femenino. Así, se muestran al público toda una heterogeneidad de apariencias, edades, etnias y razas u orientaciones sexuales, entre otros ejes.

\section{2.- Metodología}

Desde una constatación preliminar de la presencia de sujetos subalternos en OITNB, el objetivo principal de la investigación se centra en analizar cómo están representados sus personajes a partir de una serie de ejes de ejes de discriminación recogidos por la teoría interseccional, como son apariencia física, clase social, edad, etnia/raza, género, orientación sexual y religión, entre otros. A partir del mismo, se plantean las siguientes preguntas: ¿Se muestra una alteridad estereotipada o se quiebran modelos tradicionales? ¿Se construyen identidades grupales excluyentes? ¿Cómo se construye el Nosotros?, ¿y las otredades?

Para responder a estas cuestiones se hace uso del Análisis de Contenido, una técnica que nos permite detectar el "sentido latente que procede de las prácticas sociales y cognitivas" (Piñuel, 2002: 4). En otras palabras, una herramienta que posibilita el conocimiento de los mensajes explícitos y velados que se encuentran en productos culturales y relacionarlos con su contexto. Todo ello desde un enfoque cualitativo al entender que, como explica José Ignacio Ruiz Olabuénaga (2012: 233): "La flexibilidad, la adaptabilidad, la singularidad concreta, la proximidad, el pluriplanteamiento de los que hacen gala los métodos cualitativos son, todavía, más adecuados para el análisis de contenido”.

De esta forma, se toman como unidades de análisis los 36 capítulos de OITNB emitidos hasta el momento ${ }^{1}$, centrando la investigación en dos dimensiones: la alteridad y la otredad, cuya distinción cabe precisar. Con el primer término, el estudio se refiere a la representación de sujetos que se diferencian de la normatividad entendida desde la teoría feminista (varón, 
blanco, heterosexual, de clase media-alta...). Por su parte, la otredad se centra en la construcción de una oposición Nosotras-Ellos/Ellas.

Fig. 1 Tabla de dimensiones y categorías de análisis

\begin{tabular}{|c|c|}
\hline Dimensiones & Categorías \\
\hline \multirow[t]{7}{*}{ Alteridad } & Apariencia física: ausencia o presencia de belleza normativa. \\
\hline & Edad': productiva o considerada "improductiva". \\
\hline & $\begin{array}{l}\text { Diversidad funcional: ausencia/presencia de personas con enfermedades o } \\
\text { deficiencias físicas, mentales, intelectuales o sensoriales. }\end{array}$ \\
\hline & Género: hombre, mujer, transgénero. \\
\hline & Orientación sexual: bisexual, heterosexual, homosexual. \\
\hline & Etnia/Raza3: asiática, blanca, hispana, negra. \\
\hline & $\begin{array}{l}\text { Religión: ausencia (ateísmo) o presencia (budismo, catolicismo, islam, judaísmo, } \\
\text { protestantismo, otras). }\end{array}$ \\
\hline \multirow[t]{2}{*}{ Otredad } & Nosotras (grupo A) vs Ellas (grupo B, C,...) \\
\hline & Nosotras (presas) vs Institución (prisión) \\
\hline
\end{tabular}

Un análisis cuyo fin es cotejar las siguientes hipótesis: OITNB visibiliza sujetos subalternos que quiebran estereotipos tradicionalmente presentes en las ficciones televisivas. Si bien cabe precisar que reproduce otros sustentados, fundamentalmente, en la raza. En relación a esta proposición se sugiere que este eje es el más excluyente en términos grupales, junto a la edad. A su vez, se plantea que Litchfield vive una creciente sororidad a lo largo de la serie, con una progresiva alianza entre las presas frente a las instituciones.

\section{3.- Resultados}

La TED deja grandes éxitos donde las mujeres son protagonistas absolutas de la trama. Una tendencia que inicia la siempre rompedora HBO a través de Sexo en Nueva York (Sex and the City, 1998-2004) y que siguen otras producciones como Mijeres Desesperadas (Desperate Housewives, ABC, 2004-2012) o, en una versión más adolescente, Pequeñas mentirosas (Pretty Little Liars, ABC Family, 2010-). Sin embargo, Jenji Kohan da un paso más allá con OITNB pues, además de centrar la trama en personajes femeninos, apuesta por la introducción de sujetos subalternos, tantas veces inviabilizados en las ficciones. Así, la prisión de Litchfield se convierte 
en una excusa para profundizar en las realidades de las reclusas narradas mediante sus vivencias en la penitenciaría y a través de flashbacks que recapitulan sus experiencias pasadas.

También cabe indicar que uno de los elementos fundamentales para entender OITNB es que nace en un contexto marcado por la gran crisis económica que revienta en el año 2008. Una excusa para profundizar no sólo en los personajes femeninos sino en la relación de las presas con un sistema de prisiones decadente tanto a nivel individual como institucional. En definitiva, este declive se acentúa aún más con la privatización devenida en la tercera temporada.

Antes de continuar cabe advertir que, a partir de aquí, se van a producir importantes desvelamientos de la trama de las tres primeras entregas.

\subsection{Dimensión I: Alteridad}

La historia arranca desde la cotidianidad de la vida de Piper Chapman (Taylor Schilling), una joven de 32 años, emprendedora y comprometida con su novio. Su perfil, entre el WASP y el Yuppie, encaja en la normatividad física, psicológica y social. Sin embargo, su vida da un giro cuando descubre que debe entrar en prisión por un delito cometido años atrás. Este es el argumento central que invita al público a seguir las vivencias de una mujer de clase media que se aleja del prototipo carcelario. O lo que es lo mismo, "es el espectador, y los canales siempre buscan una protagonista que tenga elementos en común con la audiencia para facilitar al máximo posible el proceso de identificación que hace que te pongas en la piel de un protagonista y su circunstancia personal" (de la Torre, 2015: 134).

De esta forma, como la propia Kohan reconoce ${ }^{4}$, Chapman está pensada como un caballo de Troya. Una infiltrada que permite abrir la puerta a un mundo de mujeres altamente complejo que cuenta con una fuerte presencia de otros ejes de discriminación que se entrecruzan con el género. Es decir, clase, disfuncionalidad, edad, físico, orientación sexual, raza o religión. En definitiva, introduce numerosos perfiles alejados de la normatividad y también de unos estereotipos que suelen desarmarse mientras se profundiza en la construcción del personaje. Algo que se va a pasar a analizar a continuación.

\subsubsection{Mujeres aisladas en prisión. Ejes género, físico y orientación sexual}

Así las cosas, Chapman abre la puerta a perfiles de mujeres no normativos que usualmente quedan fuera de la televisión. En general, físicos que se alejan de la belleza estándar occidental por altura, peso o ciertos defectos -como una dentadura deteriorada-. Es decir, en muchos aspectos, se sobrepasa aquel arquetipo ligado al placer visual masculino del que hablaba Laura Mulvey (2007). Pero se va más allá, los personajes no están ligados a la simplicidad o a la 
docilidad tradicional de los roles femeninos, sino que el espectador encuentra, a la par, reclusas compasivas y crueles, débiles y fuertes, dulces y rudas, simpáticas y trascendentales. Arquetipos contradictorios que cambian según la situación y/o la evolución de la (co)protagonista.

Dentro del análisis del género, cabe destacar el caso de Sophia Burset (Laverne Cox). La peluquera de la prisión es una transgénero que termina en la cárcel por robar tarjetas de crédito para financiar su operación de reasignación de sexo. La inclusión de este personaje permite introducir problemáticas ligadas con este colectivo. Baste de ejemplo, lidiar con la incomprensión de un hijo que se niega a aceptar su cambio o con la suspensión de su tratamiento en una prisión que no lo entiende como algo necesario.

En definitiva, OITNB se convierte en un serial que apuesta por la visibilización del colectivo LGBTI, aún hoy infra-representado en la ficción 5 . Y, de nuevo, Chapman vuelve a ser el caballo de Troya para reventar la concepción binaria heterosexual. Pues la protagonista va a encontrar en prisión a la que fue su compañera de crimen y también sentimental. Un romance que no tarda en reavivarse y que pone en jaque su compromiso con su prometido. No va a ser la única, en la trama hay numerosas lesbianas como Carrie 'Big Boo' Black (Lea DeLaria), Nicky Nichols (Natasha Lyonne), Poussey Washington (Samira Wiley) o Crazy Eyes. Así como bisexuales como la citada Chapman y Lorna Morello (Yeal Stone) que mantiene un affaire puntual con Nicky.

El número se complementa con la profundización de unos personajes que huyen de la construcción simplista de híper-virilidad o el de la híper-sensualidad que tantas veces son representados en la ficción cayendo en parodia o en placer visual, muy vinculado a un lesbianismo conservador que no cuestiona la heterosexualidad normativa (Platero, 2008). Una transgresión que continúa con una representación de las relaciones que dinamita los pilares del amor romántico heterosexual presentando el amor como algo más conflictivo y menos espiritual que otras producciones. De esta forma, se ponen sobre la mesa problemáticas derivadas de la identidad y la sexualidad alejándose de la carga moral.

\subsubsection{Eje diversidad funcional}

La pluralidad de perfiles entre las reclusas alcanza también la diversidad funcional. Baste de ejemplo, Jimmy Cavanaugh (Patricia Squire) una anciana con demencia severa; Miss Rosa Cisneros (Barbara Rosenblat) una enferma de cáncer; Norma Romano (Annie Golden) una mujer tartamuda que opta por no hablar, o Taslitz (Judith Roberts) otro personaje de edad avanzada que sufre achaques como artritis o problemas de visión. Por otro lado, Crazy Eyes es diagnosticada con una enfermedad mental. Cabe señalar que, en muchas ocasiones, es un eje que ayuda a construir al personaje pero no es un condicionante para unas mujeres capaces de tomar las riendas de su vida6. 


\subsubsection{Eje religión}

Se detecta cierta tendencia a parodiar los dogmas radicales a través de personajes que utilizan las creencias con un fin personal más allá de elementos espirituales. El caso principal es el de Tiffany 'Pennsatuky' Dogget (Tatyn Manning). Dibujada desde el esperpento, la gran evangelizadora de la prisión es apoyada por un grupo de anti-abortistas que piensan que su asesinato en una clínica es ideológico -nada más alejado de la realidad-. Un perfil que se repite desde un retrato más calmado como la improvisada secta que dirige Norma, un entretenimiento inocente que termina llevando al límite a una joven depresiva. Otros cultos dibujados desde cierto humor son la santería -hispanas- o el judaísmo, cuando un grupo de reclusas se hace pasar por conversas para acceder a una mejor comida.

De hecho, esta producción está llena de referencias al ateísmo, con hueco para todo un discurso sobre la imposibilidad de creer de Chapman o un capítulo dedicado a las dudas religiosas de las reclusas a través de flashbacks. Sin embargo, también hay espacios para las creencias moderadas, como ocurre con Sister Ingalls (Beth Fowler) que va a mantener más de una charla sobre Dios con Sophia.

\subsubsection{Ejes clase y raza. El color de piel como nuevo orden social}

La elección de la prisión como escenario principal es significativo pues es capaz de aislar a las reclusas de su entorno cultural, económico y familiar. De esta forma, mujeres que, a priori, nunca se conocerían comen, duermen, trabajan y mantienen relaciones sexuales con otras sin importar el estrato social al que pertenezcan. Pues en este espacio no hay apenas diferencias más allá del limitado dinero que pueden utilizar en el pequeño puesto de la penitenciaría. Sin embargo, el eje se desdibuja pero continúa como elemento conformador de los personajes ya que sigue presente el peso del capital cultural, algo que, por ejemplo, va a servir a la protagonista para salir de más de un enredo.

Eso no quiere decir que desaparezcan los estamentos sino que el aglutinante va a ser otro. En OITNB la estratificación procede del factor racial, étnico y de procedencia -en este orden-. Así, las reclusas se agrupan en torno a tres grandes familias capaces de absorber los ejes anteriormente analizados.

\subsubsection{1 'The Suburbs'}

La familia blanca está dirigida por Galina 'Red' Reznikov (Kate Mulgrew). La rusa es la muestra de que el color de piel está por encima de procedencias donde se reúnen europeas o estadounidenses. En la primera entrega, son las encargadas de la cocina, uno de los empleos más codiciados de la prisión, y que es utilizada por Red para introducir algunos productos de contrabando -como barras de labios-. Otras ocupan diferentes trabajos que van desde chófer a mecánica. 
También cabe destacar que es el grupo más numeroso entre las (co)protagonistas y también el más heterogéneo. Así, el espectador se puede encontrar con personajes de clase baja -Morello, Pennsatuky-, media/alta -Chapman, Nicky- y trabajadora -Big Boo-. También diferentes edades -con el grupo con un mayor número de actrices que superan los 50 -. Así como con un perfil físico muy amplio -aunque el sector más joven es mayoritariamente normativo-.

Cabe destacar que es la familia con mayor número de personajes no heterosexuales, llevando además el peso de los romances entre presas encabezados por Chapman y Alex Vausse (Laura Prepon). También señalar que son el grupo más vinculado a la drogadicción.

\subsubsection{2 'The Ghetto'}

La familia afroamericana se dibuja, principalmente, desde la juventud. Y, con ello, se realza la alegría con diálogos irónicos marcados por el uso del llamado Black English o ebonics ${ }^{7}$, en una representación distendida y lozana. No obstante, cabe señalar que sus fuertes lazos hacen que no sea bien visto que se relacionen con otros grupos -como le pasa a Poussey cuando se aleja de ellas en pro de la secta de Norma-.

Dentro de esta familia, el papel de madre recae en Yvone 'Vee' Parker (Lorraine Toussaint) pero tan sólo durante la segunda temporada. Una mujer madura que va a intentar hacerse con el control de la prisión con el fin de devolver el poder a las afroamericanas -algo que la convierte en antagonista de Red y en la gran villana de esta entrega-. Tras su desaparición, el liderazgo cae en una figura más comprensiva y dedicada a los cuidados del grupo, Tasha 'Taystee' Jefferson (Danielle Brooks). Cabe señalar que la mayoría de ellas trabajan en la limpieza de la cárcel, uno de los trabajos más denostados -más adelante también en la confección de ropa interior-. Y que, además, no suelen estar ligadas a las drogas -tan sólo Poussey va a tener ciertos problemas con el alcohol que ella misma confecciona-.

Indicar también que, al final de la tercera temporada, abren la puerta a Soso, una joven de ascendencia asiática deprimida incapaz de encontrar su sitio en prisión y que, además, inicia una relación romántica con Poussey. A este respecto, destacar que esta última y Crazy Eyes van a ser las únicas homosexuales del grupo.

\subsubsection{3 'Spanish Harlem'}

La familia hispana es, sin lugar a duda, la más estereotipada. Lideradas por Gloria Mendoza (Selenis Leyva) se hacen cargo de la cocina a partir de la segunda temporada, lo que aumenta su estatus en la penitenciaría y lleva a cierta rivalidad con Red y las suyas. 
En general, se tiende a un dibujo de mujeres de clase baja, habla hispana, continuamente ligadas al amor romántico heterosexual -no hay ninguna homosexual en el grupo-, la maternidad y a las creencias místicas y religiosas. De hecho, no sólo cinco de ellas son madres sino que hay dos mujeres hispanas encinta dentro de prisión. Así, uno de los temas centrales gira en torno a que Dayanara 'Daya' Diaz (Dasha Polanco) se queda embarazada de uno de los guardias. Indicar también que se tiende a ensalzar la familia hispana como algo fundamental. Aunque, cuando se entra a profundizar en los personajes, también se problematiza este aspecto dibujando núcleos desestructurados y madres imperfectas que, en ocasiones, quieren una vida más allá de las ataduras domésticas.

\subsubsection{4 'The Others'}

El resto de la prisión tiene lazos puntuales pero se desenvuelven de forma independiente. En ocasiones el resto de reclusas hacen referencia a ellas como 'las otras'. Dentro de este grupo destacar las 'Golden Girls', que se van a analizar a continuación.

\subsubsection{Eje edad}

Litchfield cuenta con un importante número de protagonistas entre la veintena y la treintena. Sin embargo, también tiene amplio espacio para presas de mayor edad, usualmente menos frecuentes en otras producciones (Gracia, 2011). Así, se puede observar que hay un gran número de actrices que en el año 2013 -inicio de la emisión- superan los 50. Ejemplo de ello, Annie Golden (1951), Beth Fowler (1951), Constance Shulman (como Erica 'Yoga' Jones, 1958), Kate Mulgrew (1955), Judith Roberts (1934), Lea DeLaria (1958), Lori Petty (como Lolly Whitehill, 1963) o Yvette Freeman (como Irma, 1957).

Algunas de ellas están totalmente integradas en las tres grandes familias -raciales- pero también es habitual verlas entre las independientes -'The Others'- o en un cuarto grupo conocido como las 'Golden Girls'. Un clan conformado por cuatro ancianas totalmente ignoradas en la prisión: Frieda Berlin (Dale Soules), Irma, Jimmy y Taslitz. Un grupo heterogéneo que cuenta con una afroamericana ya que la edad avanzada es el único aglutinante por encima de la raza. En definitiva, un cohesivo basado en la exclusión del resto de reclusas que las infravalora e invisibiliza. Sin embargo, la profundización en los personajes que caracteriza a esta producción va a hacer que, de nuevo, se dé una vuelta de tuerca a este colectivo que se muestra, paulatinamente, cada vez más empoderado ${ }^{8}$.

\subsection{Dimensión II: Otredad}

Como se ha dicho, el espectador se introduce en la prisión a través de los ojos de la protagonista. Por ello, la primera construcción de la otredad gira en torno a un 'Nosotros, Piper', en tanto que personaje normativo, frente a 'Los Otros, Litchfield'. Así, funcionarios y reclusas conforman el escenario hostil donde ha de desenvolverse la recién llegada y que se 
encontrará con confrontaciones pequeñas - como una ofendida Red que se niega a darle de comer- y grandes -como una Pennsatucky que la amenaza constantemente-.

Sin embargo, estos enfrentamientos son una mera transición para mostrar una fotografía más amplia de la prisión. De ahí que, en la segunda entrega, las tensiones dibujadas revienten en un conflicto entre un 'Nosotras vs. Otras' en base a las citadas familias étnicas y raciales. En este caso, el mayor antagonismo va a recaer en Vee, dibujada como una mujer oscura y cruel para el resto de grupos pero también para el suyo propio -especialmente en torno a el choque con Poussey-. De hecho, va a ser uno de los pocos personajes que ancle en una visión maniquea de la trama. Pues esta producción suele huir de conceptos binarios como bondad y maldad a los que tanto nos acostumbra la ficción en pro de problemáticas mucho más complejas. Esto da paso a que mujeres al otro lado del tablero, como Pennsatucky, acaben redimiéndose cuando la narración ahonda sobre su historia.

De hecho, en numerosas ocasiones, ese 'Nosotras, familia' queda en un segundo plano en torno a un simple 'Nosotras, mujeres'. Una sororidad que puede provenir de un mal mayor-como la muerte de una presa o un sistema injusto- o de la propia complicidad femenina. Una centralidad en el cuerpo y en la sexualidad que deja conversaciones impensables para otros dramas que versan sobre los órganos sexuales femeninos o los olores y picores vaginales, entre otras.

\subsubsection{Nosotras vs Sistema}

En resumen, uno de los principales nexos de sororidad proviene de la confrontación con el sistema de prisiones. Una penitenciaría que descansa bajo los pilares del capital así como del propio patriarcado. De hecho, el 'Nosotras, presas vs. Ellos, Sistema' recae en numerosas ocasiones en un 'Nosotras, mujeres vs. Ellos, hombres'.

Como consecuencia, hay que recalcar que los personajes masculinos de la prisión suelen ser dibujados de forma superficial y, en muchas ocasiones, esperpéntica9. Esta representación, entre corrupción y mediocridad, deja situaciones altamente injustas como el hecho de liberar a Jimmy, la presa con demencia, con el fin de no tener que cuidar de ella aún sabiendo que está destinada a morir en las calles. O el pánico de Crazy Eyes con el tratamiento que dan en el pabellón dedicado a las enfermedades mentales.

Una situación que se agrava en la tercera temporada cuando la prisión es privatizada buscando rendimiento económico de las propias reclusas y que provoca situaciones degradantes. Por ejemplo, que se queden sin colchones ni biblioteca por una plaga de chinches; que la comida empeore sustancialmente, o que se reduzca el salario de los guardias introduciendo nuevo personal sin preparación alguna. Temas menores que se entremezclan con otras problemáticas de alto nivel como la impotencia de Pennsatucky que es violada repetidamente por un guardia de prisiones al que teme denunciar por el peso de su palabra. 
Aunque, tal vez, uno de los casos más significativo sea el de la propia Sophia que, a raíz de un conflicto con una presa ${ }^{10}$, comienza a vivir el acoso, el desdén o, incluso, el miedo de otras reclusas. Este hecho sirve para mostrar los estereotipos que, aún hoy en día, deben de afrontar las personas trans en su cotidianeidad, pero también los problemas institucionales que tienen que encarar. Pues todo se agrava cuando la organización de la prisión decide enviarla a aislamiento, en un principio, por su propia seguridad. Una estancia que se alarga dado que la penitenciaría no quiere que se forme un escándalo con su pésima actuación. En definitiva, una crítica a las deficiencias del sistema estadounidense de prisiones, en particular, y a las injusticias sociales, en general, que también va a ser una de las tónicas de esta producción. Tal y como resumen García y López (2014, 23-24):

Orange is the New Black muestra sin tapujos este fracaso del sueño americano. Muestra la decadencia de unos personajes que sufren el menosprecio de una sociedad posiblemente cruel con un colectivo tradicionalmente maltratado, las mujeres. Además, habla sin caer en el morbo de temas controvertidos como la bisexualidad (mostrada por medio del personaje principal), el lesbianismo, la represión sexual, el poder y la corrupción policial y las injusticias del sistema de prisiones estadounidense, más propio de países subdesarrollados.

\section{Conclusiones}

La visibilización de colectivos sociales, la problematización de la identidad femenina, así como la irreverencia temática convierten a OITNB en uno de los pocos oasis que se encuentran en el desierto conservador y androcéntrico de la ficción. Por un lado, hereda esa apuesta por la sororidad de otros productos televisivos protagonizados por mujeres, como se ha visto anteriormente, pero también esa representación de los márgenes más típica de la decadencia noir que se recupera en esta época dorada. No obstante, esta comedia dramática se aleja de la violencia simbólica que se ejerce mediante la estereotipación mediática de ciertos colectivos, de la que han sido víctimas lesbianas, negras, hispanas, personas con diversidad funcional o de edad avanzada.

Así, Jenji Kohan presenta un desafío a la normatividad simplista de la que suelen hacerse eco gran parte de las producciones televisivas. Mediante una construcción compleja de los personajes, que atiende a los diversos ejes de opresión a la que se ven sometidas, aparecen en pantalla mujeres muy distintas, con ambiciones, intereses, presencias y posiciones diferentes. Mujeres que actúan como contra-públicos subalternos, siguiendo la noción de Nancy Fraser (1990) al cuestionar las lógicas de dominación imperantes. En otras palabras, OITNB visibiliza muchas realidades que habían sido opacas hasta ahora en este tipo de dramas, pero además se aleja de posiciones victimarias apostando por la capacidad de agencia y la puesta en marcha de estrategias de supervivencia de la población de los márgenes. De esta manera, se corrobora la enunciación de la primera hipótesis sobre la visibilización de sujetos subalternos desde una representación carente de estereotipos. 
No obstante, una mirada más concentrada permite observar la presencia de los mismos en ciertas ocasiones, muy ligados a la cuestiones de raza y edad, que se constituyen como ejes de exclusión como expone la segunda hipótesis. Las familias se construyen sobre ciertos roles altamente vinculados a estereotipos étnicos, como puede ser el apego religioso, heterosexual y maternal de las hispanas; la jovialidad de las negras, o la posición cultural más elevada, en general, en las blancas. Por otro lado, es interesante señalar que el clivaje etnia/raza es el principal eje aglutinador en prisión, por encima incluso de la clase. Algo que no es ajeno al contexto estadounidense, cuya sociedad se vertebra en torno a este eje. Únicamente la edad se constituye como otro potente generador de otredades, siendo un factor de exclusión hacia aquellas consideradas distintas.

Sin embargo, cabe constatar como las diferencias entre las presas se van diluyendo en favor de una identidad colectiva que enfrenta a las reclusas contra los encargados de regir la penitenciaria, corroborando que "Litchfield vive una creciente sororidad a lo largo de la serie, con una progresiva alianza entre las presas frente a las instituciones", como manifiesta la tercera y última de las hipótesis. Ya que se yergue como un sistema carcelario que actúa como metáfora de ese entramado institucional capitalista y patriarcal, capaz de oprimir a todos aquellos grupos que considera alteridades. No obstante, las mujeres en OITNB resisten y se enfrentan a unas estructuras de dominación que las discrimina, las margina y las invisibiliza, convirtiéndose en sujetos protagonistas y ubicando en primera línea preocupaciones y cotidianeidades femeninas. Una serie que posibilita la reflexión de que, como reclama Pilar Aguilar (2012), es necesaria una mayor presencia de los grupos subalternos detrás de las cámaras, que creen escenarios simbólicos alejados de la normatividad tradicional.

\section{Bibliografía}

Aguado Peláez, Delicia (2014a). Cuando el patriarcado sobrevive al apocalipsis. Análisis de The Walking Dead. Feminismo/s, 23, 279-297.

Aguado Peláez, Delicia (2014b). Imaginario postapocalíptico en las series de televisión norteamericanas tras el 11S: Análisis de The Walking Dead (AMC, 2010-). En AEIC (ed.) Espacios de comunicación: IV Congreso Internacional de la Asociación Española de Investigación en Comunicación (pp. 1091-1100). Bilbao: AEIC.

Aguado Peláez, Delicia (2014c). La Sociedad del Riesgo en la ficción televisiva tras el 11 de Septiembre. El caso de Homeland (Showtime, 2011-) y The Walking Dead (AMC, 2010-). En Latina de Comunicación Social (ed.) Actas del VI Congreso Internacional Latina de Comunicación 
Social (pp. 1-32). La Laguna: Latina de Comunicación Social. Recuperado de http://www.revistalatinacs.org/14SLCS/2014 actas/062 Aguado.pdf

Aguado Peláez, Delicia (2015). La dominación simbólica sigue siendo cosa de hombres. Análisis de la supremacía masculina en Breaking Bad, Hijos de la Anarquía y The Walking Dead. En AECPA (ed.) Actas del XII Congreso de la Asociación de Ciencias Políticas y de la Administración (pp. 1-19). Recuperado de http://www.aecpa.es/uploads/files/modules/congress/12/papers/1195.pdf

Aguado Peláez, Delicia y Martínez García, Patricia (2015). La TV-noir de la Tercera Edad Dorada desde una perspectiva de género. Análisis de Mob City, True Detective y Fargo. En Actas del XI Congreso de Novela y Cine Negro (pendiente de publicar).

Aguilar, Pilar (1998). Mujeres de cine: retratos mágicos pero distorsionados. En Muñoz, Blanca (coord.) Medios de comunicación, mijeres y cambio cultural (pp. 221-244). Madrid: Consejería de Servicios Sociales, Dirección General de la Mujer de la Comunidad de Madrid.

Aguilar, Pilar (2012). La ficción audiovisual y la violencia contra las mujeres. Mientras tanto. Colección Meridiano de género, 1. Recuperado de http://www.mientrastanto.org/boletin$108 /$ notas/meridiano-de-genero-1

Alonso, Alba. 2012. A introduçao da interseccionalidade em Portugal: repensando as políticas de igualdade(s). Revista Crítica de Ciências Sociais, 90, 25-43. http://rccs.revues.org/1760?lang=es

Bai, Xueying (2011). Intersectionality of gender and race in Asian female images. A qualitative content analysis of U.S. advertising representation of Asian Women. Florida: University of Florida.

Cascajosa, Concepción (2005). Prime Time. Las mejores series de TV americanas, de CSI a Los Soprano. Madrid: Calamar ediciones.

Cascajosa, Concepción (2007). La caja lista. TV norteamericana de culto. Barcelona: Laertes.

Collins, Patricia (1990). Black Feminist Thought. Knowledge, consciousness and the politics of empowerment. London: Routledge.

Crenshaw, Kimberlé (1989). Demarginalizing the intersection of race and sex: A black feminist critique of antidiscrimination doctrine, feminist theory and antiracist politics. University of Chicago Legal Forum, 139-167.

Crenshaw, Kimberlé (1991). Mapping the margins: Intersectionality identity politics and violence against women of color. Stanford Law Review, 43(6), 1241-1299. 
Cruells, Marta (2015). La interseccionalidad politica: tipos y factores de entrada en la agenda politica, jurídica y de los movimientos sociales. Barcelona: Institut de Govern i Politiques Públiques de la Universitat Autónoma de Barcelona.

De la Torre, Toni (2015). Series de Culto. Barcelona: Timun Mas.

Faludi, Susan (2009). La pesadilla terrorista. Miedo y fantasía en Estados Unidos después del 11S. Barcelona: Anagrama.

Fernández, Marta y Menéndez, $M^{a}$ Isabel (2011). Lo que el ojo no ve: Renovación vs Conservadurismo en la ficción audiovisual posterior al 11-S. En Latina de Comunicación Social (ed.) Actas del III Congreso Internacional Latina de Commicación Social (pp. 1-14). La Laguna: Latina de Comunicación Social. Recuperado de http://www.revistalatinacs.org/11SLCS/actas 2011 IIICILCS/024.pdf

Fraser, Nancy (1990). Rethinking the public sphere; A contribution to the critique of actually existing democracy. Social text, 25-26, 56-80.

Fraser, Nancy (ed.) (2011). Dilemas de Justicia en el Siglo XXI. Género y globalización de Nancy Fraser. Palma de Mallorca: Universitat de les Illes Balears.

García, Javier y López, Alicia (2014). Orange is the New Black. Una visión antropológica. Revista de la comunicación de la SEECI, 35, 19-33. Recuperado de http://www.seeci.net/revista/index.php/seeci/article/view/97/114

García Martínez, Alberto Nahum (2011). El género negro se pasa a la televisión: The Wire y The Shield. Previously on: Estudios interdisciplinarios sobre la ficción televisiva en la Tercera Edad Dorada, 221-236. Recuperado de http://fama2.us.es/fco/frame/previouslyon.pdf

GLAAD (2014). Where we are on TV Report 2014. Recuperado de http:/ / www.glaad.org/wherew eareontv14

Gracia, Jorge (2011). La pantalla anciana. Algunas reflexiones sobre los derechos humanos de las personas mayores a través del cine. InterSexiones, 2, 54-84. Recuperado de http://intersexiones.es/Numero2/04GraciaIbanez.pdf

Grzanka, Patrick (2014). Intersectionality. A foundations and frontiers reader. Philadelphia: Westview Press. 
Kohan, Jenji (directora). (2013). Orange is the New Black [serie de televisión. EU.: Netflix.

Lombardo, Emanuela y Verloo, Mieke (2010). La interseccionalidad de género con otras desigualdades en la política de la Unión Europea. Revista Española de Ciencia Politica, 23, 11-30.

Mayobre, Pilar (2007). La formación de la identidad de género. Una mirada desde la filosofía. Revista Venezolana de Estudios de la Mujer, 12(28), 35-62.

Menéndez, María Isabel (2013). Medios de comunicación, género e identidad. En Díaz, Capitolina y Dema, Sandra (Eds.) Sociología y género (pp. 253-269). Madrid: Editorial Tecnos.

Mulvey, Laura (2007). El placer visual y el cine narrativo. En Cordero, K. y Sáenz. I. (comps.). Critica feminista en la teoría e historia del arte (pp. 81-93). México: Universidad Iberoamericana.

Olabuénaga, José Ignacio (2012). Metodología de la investigación cualitativa. Bilbao: Universidad de Deusto.

Pateman, Carol (1995). El contrato sexual. Barcelona: Anthropos.

Pérez Orozco, Amaia (2006). Perspectivas feministas en torno a la Economía: el caso de los Cuidados. Madrid: Consejo Económico y Social.

Piñuel, José Luís (2002). Epistemología, metodología y técnicas del análisis de contenido. Sociolinguistic Studies, 3(1), 1-42.

Platero, Raquel (Lucas) (2008). Las lesbianas en los medios de comunicación: madres, folclóricas y masculinas. En Platero, Raquel (Lucas) (Coord.) Discursos y representaciones (pp. 307338). Barcelona: Melusina.

Spivak, Gayatri (2003). ¿Puede hablar el sujeto subalterno? Revista Colombiana de Antropología, 39, 297-364.

Tous, Anna (2010). La era del drama en televisión. Perdidos, CSI: Las Vegas, El ala oeste de la Casa Blanca, Mijeres Desesperadas y House. Barcelona: UOC Press.

Variety (2014). GLAAD ReportFinds Gains in LGBT Characters on Broadcast, Cable. Variety, 1 de octubre. Recuperado de http://variety.com/2014/tv/news/glaad-report-finds-gains-inlgbt-characters-on-broadcast-cable-1201318331/ 
Young, Iris Marion (2000). La justicia y la politica de la diferencia. Madrid: Ediciones Cátedra.

\section{Notas}

1. Los 13 capítulos de cada una de sus entregas (2013, 2014 y 2015).

2. Esta investigación no está interesada en medir la edad como rango de frecuencias, sino atender a esta categoría en su dimensión de exclusión. Es decir, entendiendo que existe un sistema discriminatorio sustentado en el edadismo, en el que personas de edad avanzada son consideradas improductivas o nulas socialmente (Gracia Ibáñez, 2011). En palabras de Judith Butler, recogidas por este mismo autor, "un proceso por medio del cual se estereotipa de forma sistemática a, y en contra de, las personas mayores por el hecho de ser viejas, de la misma forma que actúan el racismo y el sexismo, en cuyos casos es debido al color de la piel o el género" (Butler y Lewis, 1973 en Gracia Ibáñez, 2011: 57).

3. En el presente estudio no se pretende entrar en los debates etnia/raza, debido a que ambos se consideran aglutinantes grupales. Así, por un lado las hispanas se unen en torno a un factor cultural -etnia-. Mientras que, por otro, Suzanne 'Crazy Eyes' Warren (Uzo Aduba), una joven afroamericana que procede de un entorno familiar y vecinal blanco se integra en prisión dentro del llamado Ghetto -color de piel-.

4. Declaraciones recogidas en: Lacey, L. (2014). 'Orange' Showrunner Jenji Kohan on Hollyw ood's Pay Inequality, 'F--- You' Money and Her 'Friends' Regrets. The Hollywood Reporter. Recuperado de http://www.hollywoodreporter.com/news/orange-is-new-blackshowrunner-723403

5. Ciertos informes como la "Guía para medios" realizado por la Alianza Gay y Lésbica contra la difamación muestra esta infra-representación en la televisión estadounidense. Así, se afirma que el número de personajes de este colectivo se limita a 65 en las grandes networks -un $44 \%$ mujeres y $26 \%$ minorías-, y a 64 en las televisiones por cable - $44 \%$ mujeres y $34 \%$ minorías-. En general, un 3,9\% de los personajes de la temporada 2014-2015 pertenecen a esta comunidad, algo superior al año anterior -aunque inferior a 2012-2013-. Además, destacan la falta de personajes transexuales en la ficción (GLAAD, 2014, Variety, 2014).

6. Baste como ejemplos, Crazy Eyes escribe con éxito una novela erótica; Jimmy planea un asesinato para equilibrar las fuerzas entre grupos, aunque resulta fallido; Miss Rosa es capaz de llevar a cabo una última fuga para terminar con su vida con dignidad; mientras Norma se convierte en la líder de una secta improvisada en prisión. 
7. Ebonics o African American Vernacular English, es un dialecto del inglés americano usualmente utilizado por las clases trabajadoras urbanas y también por clases medias afroamericanas.

8. La narración se detiene en estas 'Chicas de Oro' durante la segunda temporada, cuando Red es expulsada de la dirección de la cocina y se aleja de su clan. Derrotada y sola, la rusa es reclutada a regañadientes por estas cuatro mujeres. Paulatinamente, Red moviliza a este grupo para refundar su negocio de contrabando utilizando como tapadera el trabajo en una pequeña huerta de la cárcel. Disfrazado como terapéutico, las 'Golden Girls' van a participar de los chanchullos de la rusa. Y, con ello, a mostrar un perfil activo, fuerte e independiente. Así, Frieda cuenta que emasculó a su marido con un cuchillo de mantequilla y va a amenazar a las hispanas o Talitz, condenada por asesinato, se va a ofrecer a asesinar a Vee, algo por lo que termina en Máxima Seguridad.

9. Así, dentro de la penitenciaria se encuentra George Mendez (Pablo Schreiber) más conocido como 'Pornstache'. Un oficial que acosa continuamente a las internas, introduce drogas en la cárcel a cambio de favores sexuales y que termina perdidamente enamorado -y utilizado- por una de las reclusas. También Sam Healy (Michael Harney): un lesbófobo consejero traumatizado por su relación con su tensa esposa rusa que no habla una palabra de inglés. Pasando por Joe Caputo (Nick Sandow) al que es usual verlo masturbarse en su oficina después de la visita de las reclusas.

10. Sophia tiene un conflicto con Gloria por un tema maternal. Las tensiones terminan en una pelea donde la peluquera empuja a la hispana haciéndola caer y provocándole contusiones. A partir de ahí, Gloria y las suyas van a expandir una serie de rumores en los que se enfatiza, por primera vez en la trama, su condición transgénero. 


\section{(c) (1) (2)}

Licencia Creative Commons

Miguel Hernández Communication Journal

mhcj.es

\section{Forma de citar este artículo en las bibliografías}

Delicia Aguado Peláez y Patricia Martínez García (2015): “Otro arquetipo femenino es posible: Interseccionalidad en Orange is the New Black", en Miguel Hernández Communication Journal, n6, páginas 261 a 280. Universidad Miguel Hernández, UMH (Elche-Alicante). Recuperado el de de 20__ de: [link del artículo en mhjournal.org] 